factors, were $18.4 \%, 11.2 \%$ and $4.4 \%, 6.9 \%$ for men and women respectively.

Conclusion If individual data (eg, cohort and case-control studies) are accessible then the direct estimation of average PAF provides more realistic results. Besides, it seems that the order of important risk factors is the same in men and women except smoking.

\section{SP1-85 TROUBLED SLEEP: A CROSS-NATIONAL STUDY OF THE INFLUENCE OF AGE, HEALTH, SOCIAL AND PSYCHOSOCIAL FACTORS ON SLEEP}

doi:10.1136/jech.2011.142976n.62

\begin{abstract}
${ }^{1,2} \mathrm{G}$ Netuveli, ${ }^{1,2}{ }^{2}$ Webb, ${ }^{1,2} \mathrm{D}$ Blane. ${ }^{1}$ Imperial College London, London, UK; ${ }^{2}$ Inter-
\end{abstract} national Centre for Life Course Studies in Society and Health, UCL, London, UK

Introduction Trouble with sleep is often accepted as a part of ageing with its prevalence reported up to half of the population surveyed. However it has been persuasively argued that in healthy ageing individuals, sleep need not diminish significantly or be of poor quality and that it is the chronic diseases and functional limitations that produce sleeping problems in the elderly. The objective of this study is to compare the prevalence of sleep problems and assess the influence of age, health and psycho-social factors in a sample of European countries.

Method Data of non-institutionalised Europeans above 50 years from the Study of Health, Ageing and Retirement in Europe were used. The outcome was a binary variable based on self reported sleep problems. Explanatory variables included age, gender, and various health, social and psycho-social factors. Statistical analysis was done using multiple logistic regression and multi-level models.

Result The prevalence of sleep problems ranged from 18\% in Greece to $48 \%$ in Poland; Greece was an exception with other Southern European countries reporting more sleep problems. Age was significant factor only for women who had greater probability of sleep problems than men. Health was the major influence on sleep problems but psychosocial factors like trust in others and social factors like receiving help were also significantly associated.

Conclusions Health is the major influence on sleep with age significant only for women. However there are significant differences among European countries in the prevalence of sleep problems.

\section{SP1-86 FOLLOW-UP AND INCIDENT OUTCOMES IN A LONGITUDINAL STUDY IN BRAZIL (ELSA-BRASIL)}

doi:10.1136/jech.2011.142976n.63

S Barreto, R Ladeira, * V Passos, F Diniz, S Kelles. UFMG, Belo Horizonte/Minas Gerais, Brazil

Introduction Baseline data from Longitudinal Study of Adults Health (ELSA-Brasil), a cohort that investigates incidence and predictors of chronic diseases among 15000 civil servants aged 35-74 years from six universities in Brazil, was fully accomplished in December 2010. From now on, main challenges are: avoid loss to follow-up over time, correct identification, and classification of incident outcomes.

Methods Follow-up data collection relies on annual phone interview to identify hospitalisations and emergency department visits, 3-year examination in research centres, spontaneous participant report and linkage with available databases from human resources department of universities and death certificates. Events of interest include: acute myocardial infarction, unstable angina, heart failure, peripheral artery disease, myocardial revascularisation, resuscitated sudden death, stroke, transient ischaemic attack, incident diabetes, diabetes related complications, chronic kidney disease, cancer and all-causes mortality. Besides, intermediate outcomes such as hypertension, obesity, cognitive decline, kidney and endothelial dysfunction will be ascertained. To guarantee the uniformity of the assessment, an ELSA Outcome Committee performs a reiterated verification of the protocols, scripts and certifies periodically researchers which are in direct contact with the population under study. The Morbidity and Mortality Committee aggregates specialists from the six research centres, in order to perform events classification according to standardised protocols.

Conclusions The great number of exposures studied will allow investigate many associations with outcomes of interest. Besides that, a promising issue of this study is the biological samples and DNA bank of all participants which will also allow nested casecontrol analysis.

\section{SP1-87 PREVALENCE AND CHARACTERISTICS OF EARLY CHILDHOOD OBESITY IN TURKEY}

doi:10.1136/jech.2011.142976n.64

S Tezcan,* T Adali, E K Yigit. Hacettepe University, Ankara, Turkey

Introduction Developing societies experience a nutritional transition in line with their epidemiological transition. As a diet rich in carbohydrates and fats and poor in fibre spreads, one of the major consequences of the nutritional transition emerges: Obesity. Among all age groups, childhood obesity is especially important, since it is known that it is likely to lead to adulthood obesity; an important risk factor for cardiovascular disease and diabetes. This study focuses on early childhood (under age 5) obesity in Turkey. This emerging issue is gaining importance Turkey's public health agenda, and there are already local studies to estimate the prevalence of childhood obesity. However, no national estimates have been obtained until recently, and this study presents the findings obtained for the further analysis study of the Turkey Demographic and Health Survey, 2008.

Methods Data were obtained from the anthropometric measurements in this survey.

Results The national prevalence of childhood obesity was calculated as $5.8 \%$. It was higher in urban areas (6.7\%) than rural areas $(3.5 \%)$. It was also higher in the western region $(7.8 \%)$, wealthiest households $(11.5 \%)$; for children with at least high school graduate mothers $(9.8 \%)$, no siblings $(8.4 \%)$ and higher birth weights $(7.1 \%)$ Multiple logistic regression analysis, where being obese was the dependent variable, showed that the birth order and birth weight of the child and duration of breastfeeding were significant determinants of childhood obesity.

Conclusion The results of the study will be benefited by the health planners and administrators to combat this problem as early as possible.

\section{SP1-88 MORTALITY IN AN URBAN COHORT IN RAGAMA, SRI LANKA}

doi:10.1136/jech.2011.142976n.65

P V T S Vithanage, P A S Panapitiya, N Padmakumara, S Hemantha, K T A A Kasturiratne, A R Wickremasinghe, ${ }^{*}$ A Pathmeswaran, M J Pinidiyapathirage. Faculty of Medicine, University of Kelaniya, Ragama, Sri Lanka

Introduction The leading causes of mortality in Sri Lanka are due to chronic diseases. We describe the mortality patterns in a 35-64-yearold urban cohort resident in Ragama, Sri Lanka and followed over 3 years.

Methods A follow-up study was conducted among 2986 35-64 year olds randomly selected from the Ragama Medical Officer of Health 
area, Sri Lanka. A baseline survey was conducted from January to September 2007 and a follow-up survey was conducted from March to November 2010. Mortality data were obtained from next of kin and cause of death was verified from death certificates.

Results There were 49 deaths during 9186.46 person years of observations. Of the 49 deaths, 11 were due to myocardial infarctions, 5 were due to strokes, 5 were due to other ischaemic heart disease and the rest included 6 due to cancer and 2 due to train accidents. The increase in mortality in men occurs after 45 years and in females it is observed later on. Mortality among men was more than twice as much as females (RR 7.96 vs 3.17 per 1000 person years). All cause mortality was significantly higher in diabetics. Mortality was not associated with hypertension, dyslipidaemia, smoking, central obesity, obesity or physical activity.

Conclusions Diabetes Mellitus was significantly associated with all cause mortality. Other associations may have not been significant due to the small number of deaths.

\section{SP1-89 TENDENCY OF HORMONE REPLACEMENT THERAPY (HTR) USE IN A 18-YEAR FOLLOW-UP RETROSPECTIVE COHORT OF WOMEN IN THE VALENCIAN COMMUNITY (VC)}

doi:10.1136/jech.2011.142976n.66

\begin{abstract}
${ }^{1,2} \mathrm{R}$ Peiró-Pérez, ${ }^{1,3} \mathrm{D}$ Salas, ${ }^{1} \mathrm{G}$ Vallés, ${ }^{1,3} \mathrm{~J}$ Miranda, ${ }^{1,3} \mathrm{~J}$ Ibañez, ${ }^{1} \mathrm{~A}$ Molina-Barceló, 1,3E Pérez-Sanz.* ${ }^{1}$ Centro Superior de Investigación en Salud Pública (CSISP), Valencia, Spain; ${ }^{2}$ Ciber de Epidemiología y Salud Pública (CIBERESP), Madrid, Spain; ${ }^{3}$ Dirección General de Salud Pública, Valencian Community, Spain
\end{abstract}

Objective To described the tendency of Hormone Replacement Therapy (HTR) use in a 18-year follow-up retrospective cohort of women participating in the Valencian Community (VC), breast cancer screening program.

Methods Between 1992 and 2009, a retrospective cohort of participants in a population-based breast cancer-screening program in the VC was assessed. The study population was 683739 women, 45-69 years. Trends in current HTR use, and new and leaving users, by educational level (EL) and age group were analysed. A regression analysis by the joint point (JP) for the tendency was calculated.

Results From 1992 to 2009, the $11.73 \%(N=71827)$ of women was taken HRT, annual increases were found in the prevalence levels of HRT use to 2003 (13.5\%) and remains in 2009 (12.5\%). The peak by aged group was $20 \%$ in 2003 for the 55-59 group. The new users of HRT have increased until 1998, changing significantly, to decrease until 2009, as shows the JP regression analysis $(p<0.005)$. The university (EL) new user's peak was in 1999 (12.3\% vs 3.6\%) in the no-studies group, in 2002 (8.2\% vs $2.9 \%$ ), in 2009 (2.7\% vs $0.7 \%$ ) $(p<0.0001)$. The university leaving use peak was in 2004 (1.4\% vs $1.05 \%)$ in the no-studies, in $2009(0.4 \%$ vs $0.7 \%)$. There is a statistically significant difference in HTR users by EL, the regression analysis of the JP, shows that the trend is parallel increasing until the year 1998, and began a reduction until 2009.

Conclusions No too much impact had the WHI study in reduction of percentage of women taking HRT in our study.

\section{SP1-90 SOCI0-DEMOGRAPHIC AND GEOGRAPHIC DIFFERENCES IN HEALTHCARE RELATED OUTCOMES IN A COHORT OF DIABETES PATIENTS IN THE LAZIO REGION, ITALY}

doi:10.1136/jech.2011.142976n.67

U Kirchmayer, * V Belleudi, L Bauleo, N Agabiti, L Pinnarelli, D Fusco, M Arcà, M Davoli. Department of Epidemiology of the Regional Health Service, Lazio Region, Italy

Introduction The Italian health system guarantees care to all citizens. Previous research reported socio-demographic differences in access to evidence-based therapies, whereas studies evaluating differences in healthcare related outcomes are scarce.

Objectives Analysing socio-demographic and geographical differences in healthcare related outcomes in patients with diabetes mellitus (DM).

Methods Among all residents registered with the regional healthcare service in 2008, adult patients with DM were identified through a validated predictive model from the regional administrative health information systems (hospital discharges, drug claims, exemptions for DM). Healthcare related outcomes were hospitalisation for uncontrolled DM, short-term complications, long-term complications, and amputation of lower limb (excluding traumatic amputations). Age standardised rates were calculated by gender, geographic area and socio-economic position (SEP) of residence (only for the city of Rome, 5 levels), and RRs were calculated.

Results In 2008, 2569 cases of complications were registered among the 303.016 DM patients, with higher prevalence among males. Incidence rates varied between $0.8 / 1000$ for short-term and 4.2/1000 for long-term complications. Geographic variations were not statistically significant. Incidence of admissions for uncontrolled DM and short-term complications increased with decreasing socioeconomic position in both genders. A significant risk increase was observed among males with lowest respect to highest SEP for amputations ( $R R=5.88,95 \%$ CI 1.69 to 20.51) and for short-term complications ( $\mathrm{RR}=12.58,95 \% \mathrm{CI} 2.83$ to 55.91 ).

Conclusions In the Lazio region, complications of DM are more frequent among males. In Rome, the risk of complications is higher in DM patients belonging to lower SEP.

\section{SP1-91 NON-INVASIVE ASSESSMENT OF HEPATIC INJURY IN CHRONIC LIVER DISEASES: IMPLEMENTATION IN CLINICAL PRACTICE. A COMPARATIVE STUDY IN MANIPAL TEACHING HOSPITAL OF WESTERN NEPAL}

doi:10.1136/jech.2011.142976n.68

${ }^{1}$ A Mittal, ${ }^{1} \mathrm{~B}$ Sathian, ${ }^{*}{ }^{2} \mathrm{~A}$ Kumar, ${ }^{1} \mathrm{~N}$ Chandrasekharan. ${ }^{1}$ Manipal College of Medical Sciences, Pokhara, Nepal; ${ }^{2}$ College of Medicine \& JNM Hospital, West Bengal, India

Background Chronic hepatitis B and C together with alcoholic (ALD) and non-alcoholic fatty liver diseases (NAFLD) represent the major forms of progressive liver disease.

Objective To assess the extent of liver injury by estimation of biochemical parameters and its significance in clinical practice.

Materials and Methods It was a retrospective study carried out from the data maintained in the Department of Biochemistry of the Manipal Teaching Hospital, Pokhara, Nepal between 1 June 2009 and 31 July 2010. Descriptive statistics and testing of hypothesis were used for the analysis using SPSS 16 software.

Result Of the 653 subjects, $48.5 \%$ were controls, $19.6 \%$ ALD, Viral Hepatitis $18.4 \%$ and $13.5 \%$ had NAFLD. In cases of viral hepatitis, mean values of aspartate aminotransferase and alanine aminotransferase were markedly increased as compared to controls. However, in alcoholic patients, ALT level is usually elevated to a lesser degree than AST level. The mean value of AST was markedly increased in comparison to ALT leading to significantly higher AST/ ALT ratio 1.59 with CI (1.49 to 1.69) ( $p=0.001)$. In NAFLD patients, there was mild to moderate elevation in serum levels of AST, ALT or both. The mean value of the ratio of AST/ALT was $1.03 \pm 0.26$ but this ratio increases as fibrosis advances, leading to a loss of its diagnostic accuracy in patients with cirrhotic non-alcoholic fatty liver disease.

Conclusion Simple, cheap and reliable non-invasive biochemical markers should be used for early stage detection of liver diseases especially the countries having low socioeconomic status like Nepal. 